\title{
A note on degenerate Hermite-Fubini numbers and polynomials
}

\section{Waseem A. Khan ${ }^{1}$, Idrees A. Khan ${ }^{2}$ and Nisar $\mathbf{K} \mathbf{S}^{3}$}

${ }^{1,2}$ Department of Mathematics, Faculty of Science, Integral University, Lucknow-226026, (India)

${ }^{3}$ Department of Mathematics, College of Arts and Science-Wadi Al dawaser, Prince Sattam bin Abdulaziz University, Riyadh region 11991, Saudi Arabia

E-mail: waseem08_khan@rediffmail.com, idrees_maths@yahoo.com, n.sooppy@psau.edu.sa

Abstract. In this paper, we introduce a new class of degenerate Hermite-Fubini numbers and polynomials and investigate some properties of these polynomials. We establish summation formulas of these polynomials by summation techniques series. Furthermore, we derive symmetric identities of degenerate Hermite-Fubini numbers and polynomials by using generating functions.

Keywords: Hermite polynomials, degenerate Hermite polynomials, degenerate Fubini polynomials, degenerate Hermite-Fubini polynomials.

2010 Mathematics Subject Classification.: 11B68, 11B83, 33C45, 33C99.

\section{Introduction}

The 2-variable Hermite Kampé de Fériet polynomials (2VHKdFP) $H_{n}(x, y)[1$, 4] are defined as

$$
H_{n}(x, y)=n ! \sum_{r=0}^{\left[\frac{n}{2}\right]} \frac{y^{r} x^{n-2 r}}{r !(n-2 r) !}
$$

It is clear that

$$
H_{n}(2 x,-1)=H_{n}\left(x, H_{n}\left(x,-\frac{1}{2}\right)=H e_{n}(x), H_{n}(x, 0)=x^{n},\right.
$$

where $H_{n}(x)$ and $H e_{n}(x)$ being ordinary Hermite polynomials.

The Hermite polynomial $H_{n}(\mathrm{x}, \mathrm{y})$ (see $([12,13])$ is defined by means of the following generating function as follows:

$$
e^{x t+y t^{2}}=\sum_{n=0}^{\infty} H_{n}(x, y) \frac{t^{n}}{n !}
$$

Recently, Khan [7] introduced degenerate Hermite polynomials by means of the following generating function as follows:

$$
(1+\lambda t)^{\frac{x}{\lambda}}\left(1+\lambda t^{2}\right)^{\frac{y}{\lambda}}=\sum_{n=0}^{\infty} H_{n}(x, y ; \lambda) \frac{t^{n}}{n !} .
$$

Note that

$$
\lim _{\lambda \longrightarrow 0}(1+\lambda t)^{\frac{x}{\lambda}}=e^{x t} .
$$

It is evident that (1.3) reduces to (1.2). That is $H_{n}(x, y)$ limiting case of $H_{n}(x, y ; \lambda)$, when $\lim _{\lambda \longrightarrow 0}$. 
The explicit representation of degenerate Hermite polynomials $H_{n}(x, y ; \lambda)$ as follows:

$$
H_{n}(x, y ; \lambda)=n ! \sum_{r=0}^{\left[\frac{n}{2}\right]} \frac{\lambda^{n-r}\left(\frac{x}{\lambda}\right)_{n-2 r}\left(\frac{y}{\lambda}\right)_{r}}{r !(n-2 r) !}
$$

For $\lambda \in \mathbb{C}$, Carlitz introduced the degenerate Bernoulli polynomials given by the generating function

$$
\frac{t}{(1+\lambda t)^{\frac{1}{\lambda}}-1}(1+\lambda t)^{\frac{x}{\lambda}}=\sum_{n=0}^{\infty} \beta_{n}(x ; \lambda) \frac{t^{n}}{n !}, \quad(\text { see }[3,8,9,10,11])
$$

so that

$$
\beta_{n}(x ; \lambda)=\sum_{n=0}^{m}\left(\begin{array}{l}
n \\
m
\end{array}\right) \beta_{m}(\lambda)\left(\frac{x}{\lambda}\right)_{n-m} .
$$

When $x=0, \beta_{n}(\lambda)=\beta_{n}(0 ; \lambda)$ are called the degenerate Bernoulli numbers.

From (1.5), we note that

$$
\begin{gathered}
\sum_{n=0}^{\infty} \lim _{\lambda \longrightarrow 0} \beta_{n}(x ; \lambda) \frac{t^{n}}{n !}=\lim _{\lambda \longrightarrow 0} \frac{t}{(1+\lambda t)^{\frac{1}{\lambda}}-1}(1+\lambda t)^{\frac{x}{\lambda}} \\
=\frac{t}{e^{t}-1} e^{x t}=\sum_{n=0}^{\infty} B_{n}(x) \frac{t^{n}}{n !}
\end{gathered}
$$

where $B_{n}(x)$ are called the Bernoulli polynomials (see [1-15]). (see $[2])$ :

Geometric polynomials (also known as Fubini polynomials) are defined as follows

$$
F_{n}(x)=\sum_{k=0}^{n}\left\{\begin{array}{l}
n \\
k
\end{array}\right\} k ! x^{k}
$$

where $\left\{\begin{array}{l}n \\ k\end{array}\right\}$ is the Stirling number of the second kind (see [5]).

For $x=1$ in (1.8), we get $n^{\text {th }}$ Fubini number (ordered Bell number or geometric number) $F_{n}[2,5,6,15]$ is defined by

$$
F_{n}(1)=F_{n}=\sum_{k=0}^{n}\left\{\begin{array}{l}
n \\
k
\end{array}\right\} k !
$$

The exponential generating functions of geometric polynomials is given by (see [2]):

$$
\frac{1}{1-x\left(e^{t}-1\right)}=\sum_{n=0}^{\infty} F_{n}(x) \frac{t^{n}}{n !}
$$

and related to the geometric series (see [2]):

$$
\left(x \frac{d}{d x}\right)^{m} \frac{1}{1-x}=\sum_{k=0}^{\infty} k^{m} x^{k}=\frac{1}{1-x} F_{m}\left(\frac{x}{1-x}\right),|x|<1 .
$$

Let us give a short list of these polynomials and numbers as follows:

$F_{0}(x)=1, F_{1}(x)=x, F_{2}(x)=x+2 x^{2}, F_{3}(x)=x+6 x^{2}+6 x^{3}, F_{4}(x)=x+14 x^{2}+36 x^{3}+24 x^{4}$, and

$$
F_{0}=1, F_{1}=1, F_{2}=3, F_{3}=13, F_{4}=75 .
$$

Geometric and exponential polynomials are connected by the relation (see [2]):

$$
F_{n}(x)=\int_{0}^{\infty} \phi_{n}(x) e^{-\lambda} d \lambda
$$


In (2016), Khan [7] introduced two variable degenerate Hermite-poly-Bernoulli polynomials is defined by means of the following generating function:

$$
\frac{\operatorname{Li}_{k}\left(1-e^{-t}\right)}{(1+\lambda t)^{\frac{1}{\lambda}}-1}(1+\lambda t)^{\frac{x}{\lambda}}\left(1+\lambda t^{2}\right)^{\frac{y}{\lambda}}=\sum_{n=0}^{\infty} H \beta_{n}^{(k)}(x, y ; \lambda) \frac{t^{n}}{n !},
$$

so that

$$
{ }_{H} \beta_{n}^{(k)}(x, y ; \lambda)=\sum_{m=0}^{n}\left(\begin{array}{l}
n \\
m
\end{array}\right) \beta_{n-m}^{(k)}(\lambda) H_{m}(x, y ; \lambda) .
$$

The object of this paper, we consider generating functions for degenerate HermiteFubini numbers and polynomials and give some properties of these numbers and polynomials. We derive summation formulas of degenerate Hermite-Fubini numbers and polynomials and we construct a symmetric identities of degenerate Hermite-Fubini numbers and polynomials by using generating functions.

\section{Degenerate Hermite-Fubini numbers and polynomials}

In this section, we define three-variable degenerate Hermite-Fubini polynomials and obtain some basic properties which gives us new formula for ${ }_{H} F_{n, \lambda}(x, y ; z)$ as follows:

We introduce 3-variable degenerate Hermite-Fubini polynomials by means of the following generating function:

$$
\frac{1}{1-z\left((1+\lambda t)^{\frac{1}{\lambda}}-1\right)}(1+\lambda t)^{\frac{x}{\lambda}}\left(1+\lambda t^{2}\right)^{\frac{y}{\lambda}}=\sum_{n=0}^{\infty}{ }_{H} F_{n, \lambda}(x, y ; z) \frac{t^{n}}{n !} .
$$

When $x=y=0 . z=1$ in (2.1), we have

$$
{ }_{H} F_{n, \lambda}(0,0 ; z)=F_{n, \lambda}(z),{ }_{H} F_{n, \lambda}(0,0 ; 1)=F_{n, \lambda} .
$$

Not that $\lim _{\lambda \longrightarrow 0}{ }_{H} F_{n, \lambda}(x, y ; z)={ }_{H} F_{n}(x, y ; z)$.

On setting $y=0$ in (2.1), we obtain 2-variable Fubini polynomials which is defined by Kim et al. [9].

$$
\frac{1}{1-z\left((1+\lambda t)^{\frac{1}{\lambda}}-1\right)}(1+\lambda t)^{\frac{x}{\lambda}}=\sum_{n=0}^{\infty} F_{n, \lambda}(x ; z) \frac{t^{n}}{n !} .
$$

Theorem 2.1. For $n \geq 0$, we have

$$
{ }_{H} F_{n, \lambda}(x, y ; z)=\sum_{m=0}^{n}\left(\begin{array}{c}
n \\
r
\end{array}\right) F_{n-m, \lambda}(z) H_{m}(x, y ; \lambda) .
$$

Proof. Using definition (2.1), we have

$$
\begin{gathered}
\sum_{n=0}^{\infty} H_{n, \lambda}(x, y ; z) \frac{t^{n}}{n !}=\frac{1}{1-z\left((1+\lambda t)^{\frac{1}{\lambda}}-1\right)}(1+\lambda t)^{\frac{x}{\lambda}}\left(1+\lambda t^{2}\right)^{\frac{y}{\lambda}} \\
=\sum_{n=0}^{\infty} F_{n, \lambda}(z) \frac{t^{n}}{n !} \sum_{m=0}^{\infty} H_{m}(x, y ; \lambda) \frac{t^{m}}{m !} \\
=\sum_{n=0}^{\infty}\left(\sum_{m=0}^{n}\left(\begin{array}{c}
n \\
r
\end{array}\right) F_{n-m, \lambda}(z) H_{m}(x, y ; \lambda)\right) \frac{t^{n}}{n !} .
\end{gathered}
$$


Comparing the coefficients of $\frac{t^{n}}{n !}$ yields (2.3).

Theorem 2.2. For $n \geq 0$, we have

$$
{ }_{H} F_{n, \lambda}(x, y ; z)=\sum_{r=0}^{n}\left(\begin{array}{c}
n \\
r
\end{array}\right) H_{n-r}(x, y ; \lambda) \sum_{k=0}^{r} z^{k} k ! S_{2}(r, k) .
$$

Proof. Using definition (2.1), we have

$$
\begin{aligned}
\sum_{n=0}^{\infty} H_{n, \lambda} F_{n,}(x, y ; z) \frac{t^{n}}{n !}=\frac{1}{1-z\left((1+\lambda t)^{\frac{1}{\lambda}}-1\right)}(1+\lambda t)^{\frac{x}{\lambda}}\left(1+\lambda t^{2}\right)^{\frac{y}{\lambda}} \\
=(1+\lambda t)^{\frac{x}{\lambda}}\left(1+\lambda t^{2}\right)^{\frac{y}{\lambda}} \sum_{k=0}^{\infty} z^{k}\left((1+\lambda t)^{\frac{1}{\lambda}}-1\right)^{k} \\
=(1+\lambda t)^{\frac{x}{\lambda}}\left(1+\lambda t^{2}\right)^{\frac{y}{\lambda}} \sum_{k=0}^{\infty} z^{k} \sum_{n=k}^{\infty} S_{2}(r, k) \frac{t^{r}}{r !} \\
=\sum_{n=0}^{\infty} H_{n}(x, y ; \lambda) \frac{t^{n}}{n !}\left(\sum_{r=0}^{\infty} \sum_{k=0}^{r} z^{k} k ! S_{2}(r, k) \frac{t^{r}}{r !}\right) \\
\text { L.H.S }=\sum_{n=0}^{\infty}\left(\sum_{r=0}^{n}\left(\begin{array}{c}
n \\
r
\end{array}\right) H_{n-r}(x, y ; \lambda) \sum_{k=0}^{r} z^{k} k ! S_{2}(r, k)\right) \frac{t^{n}}{n !} .
\end{aligned}
$$

Equating the coefficients of $\frac{t^{n}}{n !}$ in both sides, we get (2.4).

Theorem 2.3. For $n \geq 0$, the following formula for degenerate Hermite-Fubini polynomials holds true:

$$
\frac{1}{1-z} \sum_{m=0}^{n}\left(\begin{array}{c}
n \\
m
\end{array}\right) F_{m, \lambda}\left(\frac{z}{1-z}\right) H_{n-m, \lambda}(x, y)=\sum_{r=0}^{n}\left(\begin{array}{c}
n \\
r
\end{array}\right) \sum_{k=0}^{\infty} z^{k}(k)_{r, \lambda} H_{n-r, \lambda}(x, y) .
$$

Proof. We begin with the definition (2.1) and write

$$
\begin{aligned}
& \qquad \begin{array}{l}
\sum_{n=0}^{\infty} H F_{n, \lambda}(x, y ; z) \frac{t^{n}}{n !}=\frac{1}{1-z\left((1+\lambda t)^{\frac{1}{\lambda}}-1\right)}(1+\lambda t)^{\frac{x}{\lambda}}\left(1+\lambda t^{2}\right)^{\frac{y}{\lambda}} \\
\text { Let } \frac{1}{1-z}\left(\frac{1}{1-\frac{z}{1-z}(1+\lambda t)^{\frac{1}{\lambda}}-1}\right)=\frac{1}{1-z(1+\lambda t)^{\frac{1}{\lambda}}}=\sum_{k=0}^{\infty} z^{k}(1+\lambda t)^{\frac{k}{\lambda}} \\
=\sum_{r=0}^{\infty}\left(\sum_{k=0}^{\infty} z^{k}(k)_{r, \lambda}\right) \frac{t^{r}}{r !} \\
\left.\qquad \begin{array}{rl}
\sum_{n=0}^{\infty} H F_{n, \lambda}(x, y ; z) \frac{t^{n}}{n !} & =\sum_{r=0}^{\infty}\left(\sum_{k=0}^{\infty} z^{k}(k)_{r, \lambda}\right) \frac{t^{r}}{r !}\left(\sum_{n=0}^{\infty} H_{n, \lambda}(x, y) \frac{t^{n}}{n !}\right.
\end{array}\right) \\
=\sum_{n=0}^{\infty}\left(\begin{array}{c}
n \\
\left.\sum_{r=0}^{n}\left(\begin{array}{c}
n \\
r
\end{array}\right) \sum_{k=0}^{\infty} z^{k}(k)_{r, \lambda} H_{n-r, \lambda}(x, y)\right) \frac{t^{n}}{n !} .
\end{array}\right.
\end{array}
\end{aligned}
$$

Now, we observe that, by (2.6), we get

$$
\frac{1}{1-z}\left(\frac{1}{1-\frac{z}{1-z}(1+\lambda t)^{\frac{1}{\lambda}}-1}\right)=\frac{1}{1-z} \sum_{n=0}^{\infty} F_{n, \lambda}\left(\frac{z}{1-z}\right) \frac{t^{n}}{n !}
$$


Then, we have

$$
\begin{gathered}
\sum_{n=0}^{\infty}{ }_{H} F_{n, \lambda}(x, y ; z) \frac{t^{n}}{n !}=\frac{1}{1-z} \sum_{m=0}^{\infty} F_{m, \lambda}\left(\frac{z}{1-z}\right) \frac{t^{m}}{m !}\left(\sum_{n=0}^{\infty} H_{n, \lambda}(x, y) \frac{t^{n}}{n !}\right) \\
=\frac{1}{1-z} \sum_{n=0}^{\infty}\left(\sum_{m=0}^{n}\left(\begin{array}{c}
n \\
m
\end{array}\right) F_{m, \lambda}\left(\frac{z}{1-z}\right) H_{n-m, \lambda}(x, y)\right) \frac{t^{n}}{n !} .
\end{gathered}
$$

Comparing the coefficients of $\frac{t^{n}}{n !}$ in equation (2.7) and (2.8), we get (2.5).

Theorem 2.4. For $n \geq 0$, the following formula for degenerate Hermite-Fubini polynomials holds true:

$$
H_{n, \lambda}(x, y)={ }_{H} F_{n, \lambda}(x, y ; z)-z_{H} F_{n, \lambda}(x+1, y ; z)+z_{H} F_{n, \lambda}(x, y ; z) .
$$

Proof. We begin with the definition (2.1) and write

$$
\begin{aligned}
& (1+\lambda t)^{\frac{x}{\lambda}}\left(1+\lambda t^{2}\right)^{\frac{y}{\lambda}}=\frac{1-z\left((1+\lambda t)^{\frac{1}{\lambda}}-1\right)}{1-z\left((1+\lambda t)^{\frac{1}{\lambda}}-1\right)}(1+\lambda t)^{\frac{x}{\lambda}}\left(1+\lambda t^{2}\right)^{\frac{y}{\lambda}} \\
= & \frac{(1+\lambda t)^{\frac{x}{\lambda}}\left(1+\lambda t^{2}\right)^{\frac{y}{\lambda}}}{1-z\left((1+\lambda t)^{\frac{1}{\lambda}}-1\right)}-\frac{z\left((1+\lambda t)^{\frac{1}{\lambda}}-1\right)}{1-z\left((1+\lambda t)^{\frac{1}{\lambda}}-1\right)}(1+\lambda t)^{\frac{x}{\lambda}}\left(1+\lambda t^{2}\right)^{\frac{y}{\lambda}} .
\end{aligned}
$$

Then using the definition of Kampé de Fériet generalization of the degenerate Hermite polynomials $H_{n, \lambda}(x, y)(1.3)$ and (2.1), we have

$$
\sum_{n=0}^{\infty} H_{n, \lambda}(x, y) \frac{t^{n}}{n !}=\sum_{n=0}^{\infty}\left[{ }_{H} F_{n, \lambda}(x, y ; z)-z_{H} F_{n, \lambda}(x+1, y ; z)+z_{H} F_{n, \lambda}(x, y ; z)\right] \frac{t^{n}}{n !} .
$$

Finally, comparing the coefficients of $\frac{t^{n}}{n !}$, we get (2.9).

Theorem 2.5. For $n \geq 0$ and $z_{1} \neq z_{2}$, the following formula for degenerate HermiteFubini polynomials holds true:

$$
\begin{gathered}
\sum_{k=0}^{n}\left(\begin{array}{c}
n \\
k
\end{array}\right){ }_{H} F_{n-k, \lambda}\left(x_{1}, y_{1} ; z_{1}\right)_{H} F_{k, \lambda}\left(x_{2}, y_{2} ; z_{2}\right) \\
=\frac{z_{2 H} F_{n, \lambda}\left(x_{1}+x_{2}, y_{1}+y_{2} ; z_{2}\right)-z_{1 H} F_{n, \lambda}\left(x_{1}+x_{2}, y_{1}+y_{2} ; z_{1}\right)}{z_{2}-z_{1}} .
\end{gathered}
$$

Proof. The products of (2.1) can be written as

$$
\begin{gathered}
\sum_{n=0}^{\infty} \sum_{k=0}^{\infty}{ }_{H} F_{n}\left(x_{1}, y_{1} ; z_{1}\right) \frac{t^{n}}{n !} H_{k} F_{k}\left(x_{2}, y_{2} ; z_{2}\right) \frac{t^{k}}{k !}=\frac{(1+\lambda t)^{\frac{x_{1}}{\lambda}}\left(1+\lambda t^{2}\right)^{\frac{y_{1}}{\lambda}}}{1-z_{1}\left((1+\lambda t)^{\frac{1}{\lambda}}-1\right)} \frac{(1+\lambda t)^{\frac{x_{2}}{\lambda}}\left(1+\lambda t^{2}\right)^{\frac{y_{2}}{\lambda}}}{1-z_{2}\left((1+\lambda t)^{\frac{1}{\lambda}}-1\right)} \\
\sum_{n=0}^{\infty}\left(\sum_{k=0}^{n}\left(\begin{array}{c}
n \\
k
\end{array}\right){ }_{H} F_{n-k}\left(x_{1}, y_{1} ; z_{1}\right)_{H} F_{k}\left(x_{2}, y_{2} ; z_{2}\right)\right) \frac{t^{n}}{n !} \\
=\frac{z_{2}}{z_{2}-z_{1}} \frac{(1+\lambda t)^{\frac{x_{1}+x_{2}}{\lambda}}\left(1+\lambda t^{2}\right)^{\frac{y_{1}+y_{2}}{\lambda}}}{1-z_{1}\left((1+\lambda t)^{\frac{1}{\lambda}}-1\right)}-\frac{z_{1}}{z_{2}-z_{1}} \frac{(1+\lambda t)^{\frac{x_{1}+x_{2}}{\lambda}}\left(1+\lambda t^{2}\right)^{\frac{y_{1}+y_{2}}{\lambda}}}{1-z_{2}\left((1+\lambda t)^{\frac{1}{\lambda}}-1\right)} \\
=\left(\frac{z_{2 H} F_{n}\left(x_{1}+x_{2}, y_{1}+y_{2} ; z_{2}\right)-z_{1 H} F_{n}\left(x_{1}+x_{2}, y_{1}+y_{2} ; z_{1}\right)}{z_{2}-z_{1}}\right) \frac{t^{n}}{n !} .
\end{gathered}
$$

By equating the coefficients of $\frac{t^{n}}{n !}$ on both sides, we get (2.10). 
Theorem 2.6. For $n \geq 0$, the following formula for degenerate Hermite-Fubini polynomials holds true:

$$
z_{H} F_{n, \lambda}(x+1, y ; z)=(1+z)_{H} F_{n, \lambda}(x, y ; z)-H_{n, \lambda}(x, y) .
$$

Proof. From (2.1), we have

$$
\begin{gathered}
\sum_{n=0}^{\infty}\left[{ }_{H} F_{n, \lambda}(x+1, y ; z)-{ }_{H} F_{n, \lambda}(x, y ; z)\right] \frac{t^{n}}{n !}=\frac{(1+\lambda t)^{\frac{x}{\lambda}}\left(1+\lambda t^{2}\right)^{\frac{y}{\lambda}}}{1-z\left((1+\lambda t)^{\frac{1}{\lambda}}-1\right)}\left((1+\lambda t)^{\frac{1}{\lambda}}-1\right) \\
=\frac{1}{z}\left[\frac{(1+\lambda t)^{\frac{x}{\lambda}}\left(1+\lambda t^{2}\right)^{\frac{y}{\lambda}}}{1-z\left((1+\lambda t)^{\frac{1}{\lambda}}-1\right)}-(1+\lambda t)^{\frac{x}{\lambda}}\left(1+\lambda t^{2}\right)^{\frac{y}{\lambda}}\right] \\
=\frac{1}{z} \sum_{n=0}^{\infty}\left[{ }_{H} F_{n}(x, y ; z)-H_{n}(x, y)\right] \frac{t^{n}}{n !}
\end{gathered}
$$

Comparing the coefficients of $\frac{t^{n}}{n !}$ on both sides, we obtain (2.11).

Remark 2.3. On setting $x=y=0$ and $x=-1$ in Theorem 2.6, we find

$$
z_{H} F_{n, \lambda}(1,0 ; z)=(1+z)_{H} F_{n, \lambda}(0,0 ; z)
$$

and

$$
z_{H} F_{n \lambda}(0,0 ; z)=(1+z)_{H} F_{n, \lambda}(-1,0 ; z)-(-\lambda)^{n}\left(\frac{1}{\lambda}\right)_{n} .
$$

Theorem 2.7. For $n \geq 0, p, q \in \mathbb{R}$, the following formula for degenerate HermiteFubini polynomials holds true:

$$
\begin{gathered}
H F_{n, \lambda}(p x, q y ; z) \\
=n ! \sum_{k=0}^{n} \sum_{j=0}^{\left[\frac{k}{2}\right]} \lambda^{k-j}{ }_{H} F_{n-k, \lambda}(x, y ; z)\left(\frac{(p-1) x}{\lambda}\right)_{k-2 j}\left(\frac{(q-1) y}{\lambda}\right)_{j} \frac{1}{(n-k-2 j) ! j !} .
\end{gathered}
$$

Proof. Rewrite the generating function (2.1), we have

$$
\begin{aligned}
& \sum_{n=0}^{\infty} H_{n} F_{n}(p x, q y ; z) \frac{t^{n}}{n !}=\frac{1}{1-z\left((1+\lambda t)^{\frac{1}{\lambda}}-1\right)}(1+\lambda t)^{\frac{x}{\lambda}}\left(1+\lambda t^{2}\right)^{\frac{y}{\lambda}}(1+\lambda t)^{\frac{(p-1) x}{\lambda}}\left(1+\lambda t^{2}\right)^{\frac{(q-1) y}{\lambda}} \\
& =\left(\sum_{n=0}^{\infty} H F_{n, \lambda}(x, y ; z) \frac{t^{n}}{n !}\right)\left(\sum_{k=0}^{\infty}\left(\frac{(p-1) x}{\lambda}\right)_{k} \lambda^{k} \frac{t^{k}}{k !}\right)\left(\sum_{j=0}^{\infty}\left(\frac{(q-1) y}{\lambda}\right)_{j} \lambda^{j} \frac{t^{2 j}}{j !}\right) \\
& =\left(\sum_{n=0}^{\infty} H F_{n, \lambda}(x, y ; z) \frac{t^{n}}{n !}\right)\left(\sum_{k=0}^{\infty} \sum_{j=0}^{\infty}\left(\frac{(p-1) x}{\lambda}\right)_{k}\left(\frac{(q-1) y}{\lambda}\right)_{j} \lambda^{k+j} \frac{t^{k+2 j}}{k ! j !}\right)
\end{aligned}
$$

Replacing $k$ by $k-2 j$ in above equation, we have

L.H.S. $=\left(\sum_{n=0}^{\infty} H_{n} F_{n}(x, y ; z) \frac{t^{n}}{n !}\right)\left(\sum_{k=2 j}^{\infty} \lambda^{k-j}\left(\frac{(p-1) x}{\lambda}\right)_{k-2 j}\left(\frac{(q-1) y}{\lambda}\right)_{j} \frac{t^{k}}{(k-2 j) ! j !}\right)$.

Again replacing $n$ by $n-k$ in above equation, we have

L.H.S. $=\sum_{n=0}^{\infty} \sum_{k=0}^{n} \sum_{j=0}^{\left[\frac{k}{2}\right]} \lambda^{k-j}{ }_{H} F_{n-k, \lambda}(x, y ; z)\left(\frac{(p-1) x}{\lambda}\right)_{k-2 j}\left(\frac{(q-1) y}{\lambda}\right)_{j} \frac{t^{n}}{(n-k-2 j) ! j ! k !}$.

Finally, equating the coefficients of $t^{n}$ on both sides, we acquire the result (2.14). 
Theorem 2.8. For $n \geq 0$, the following formula for degenerate Hermite-Fubini polynomials holds true:

$$
{ }_{H} F_{n, \lambda}(x+r, y ; z)=\sum_{l=0}^{n}\left(\begin{array}{l}
n \\
l
\end{array}\right) H_{n-l, \lambda}(x, y) \sum_{k=0}^{l} z^{k} k ! S_{2, \lambda}(l+r, k+r) .
$$

Proof. Replacing $x$ by $x+r$ in (2.1), we have

$$
\begin{aligned}
& \sum_{n=0}^{\infty}{ }_{H} F_{n, \lambda}(x+r, y ; z) \frac{t^{n}}{n !}=\frac{(1+\lambda t)^{\frac{x+r}{\lambda}}\left(1+\lambda t^{2}\right)^{\frac{y}{\lambda}}}{1-z\left((1+\lambda t)^{\frac{1}{\lambda}}-1\right)} \\
= & (1+\lambda t)^{\frac{x}{\lambda}}\left(1+\lambda t^{2}\right)^{\frac{y}{\lambda}}(1+\lambda t)^{\frac{r}{\lambda}} \sum_{k=0}^{\infty} z^{k}\left((1+\lambda t)^{\frac{1}{\lambda}}-1\right)^{k} \\
= & (1+\lambda t)^{\frac{x}{\lambda}}\left(1+\lambda t^{2}\right)^{\frac{y}{\lambda}}(1+\lambda t)^{\frac{r}{\lambda}} \sum_{k=0}^{\infty} z^{k} \sum_{l=k}^{\infty} k ! S_{2}(l, k) \frac{t^{l}}{l !} \\
= & \sum_{n=0}^{\infty} H_{n, \lambda}(x, y) \frac{t^{n}}{n !} \sum_{l=0}^{\infty} z^{k} \sum_{k=0}^{l} k ! S_{2, \lambda}(l+r, k+r) \frac{t^{l}}{l !} .
\end{aligned}
$$

Replacing $n$ by $n-l$ in above equation, we get

$$
\sum_{n=0}^{\infty}{ }_{H} F_{n, \lambda}(x+r, y ; z) \frac{t^{n}}{n !}=\sum_{n=0}^{\infty}\left(\sum_{l=0}^{n}\left(\begin{array}{c}
n \\
l
\end{array}\right) H_{n-l, \lambda}(x, y) \sum_{k=0}^{l} z^{k} k ! S_{2, \lambda}(l+r, k+r)\right) \frac{t^{n}}{n !} .
$$

Comparing the coefficients of $\frac{t^{n}}{n !}$ in both sides, we get (2.15).

\section{Summation Formulae for degenerate Hermite-Fubini polynomials}

First, we prove the following result involving the degenerate Hermite-Fubini polynomials ${ }_{H} F_{n, \lambda}(x, y ; z)$ by using series rearrangement techniques and considered its special case:

Theorem 3.1. The following summation formula for degenerate Hermite-Fubini polynomials ${ }_{H} F_{n}(x, y ; z)$ holds true:

$$
\begin{gathered}
{ }_{H} F_{n, \lambda}(u, v ; z)_{H} F_{m, \lambda}(U, V ; Z)=\sum_{r, k=0}^{n, m}\left(\begin{array}{c}
n \\
r
\end{array}\right)\left(\begin{array}{c}
m \\
k
\end{array}\right) H_{r, \lambda}(u-x, v-y)_{H} F_{n-r, \lambda}(x, y ; z) \\
\times H_{k, \lambda}(U-X, V-Y)_{H} F_{m-k, \lambda}(X, Y ; Z) .
\end{gathered}
$$

Proof. Consider the product of the degenerate Hermite-Fubini polynomials, we can be written as generating function (2.1) in the following form:

$$
\begin{gathered}
\frac{1}{1-z\left((1+\lambda t)^{\frac{1}{\lambda}}-1\right)}(1+\lambda t)^{\frac{x}{\lambda}}\left(1+\lambda t^{2}\right)^{\frac{y}{\lambda}} \frac{1}{1-Z\left((1+\lambda T)^{\frac{1}{\lambda}}-1\right)}(1+\lambda T)^{\frac{x}{\lambda}}\left(1+\lambda T^{2}\right)^{\frac{y}{\lambda}} \\
=\sum_{n=0}^{\infty} H_{n, \lambda}(x, y ; z) \frac{t^{n}}{n !} \sum_{m=0}^{\infty}{ }_{H} F_{m, \lambda}(X, Y ; Z) \frac{T^{m}}{m !} .
\end{gathered}
$$

Replacing $x$ by $u, y$ by $v, X$ by $U$ and $Y$ by $V$ in (3.2) and equating the resultant to itself,

$$
\begin{gathered}
\sum_{n=0}^{\infty} \sum_{m=0}^{\infty}{ }_{H} F_{n, \lambda}(u, v ; z)_{H} F_{m, \lambda}(U, V ; Z) \frac{t^{n}}{n !} \frac{T^{m}}{m !} \\
=(1+\lambda t)^{\frac{u-x}{\lambda}}\left(1+\lambda t^{2}\right)^{\frac{v-y}{\lambda}}(1+\lambda T)^{\frac{U-X}{\lambda}}\left(1+\lambda T^{2}\right)^{\frac{V-Y}{\lambda}}
\end{gathered}
$$




$$
\times \sum_{n=0}^{\infty} \sum_{m=0}^{\infty}{ }_{H} F_{n, \lambda}(x, y ; z)_{H} F_{m, \lambda}(X, Y ; Z) \frac{t^{n}}{n !} \frac{T^{m}}{m !}
$$

which on using the generating function [14] in the r.h.s., becomes

$$
\begin{gathered}
\sum_{n=0}^{\infty} \sum_{m=0}^{\infty}{ }_{H} F_{n, \lambda}(u, v ; z)_{H} F_{m, \lambda}(U, V ; Z) \frac{t^{n}}{n !} \frac{T^{m}}{m !} \\
=\sum_{n, r=0}^{\infty} H_{r, \lambda}(u-x, v-y)_{H} F_{n, \lambda}(x, y ; z) \frac{t^{n+r}}{n ! r !} \sum_{m, k=0}^{\infty} H_{k, \lambda}(U-X, V-Y)_{H} F_{m, \lambda}(X, Y ; Z) \frac{T^{m+k}}{m ! k !} .
\end{gathered}
$$

Finally, replacing $n$ by $n-r$ and $m$ by $m-k$ and using the lemma [14] in the r.h.s. of the above equation and then equating the coefficients of like powers of $t$ and T, we get assertion (3.1) of Theorem 3.1.

Theorem 3.2. The following summation formula for degenerate Hermite-Fubini polynomials ${ }_{H} F_{n}(x, y ; z)$ holds true:

$$
{ }_{H} F_{n, \lambda}(x+w, y+u ; z)=\sum_{s=0}^{n}\left(\begin{array}{c}
n \\
s
\end{array}\right){ }_{H} F_{n-s, \lambda}(x, y ; z) H_{s, \lambda}(w, u) .
$$

Proof. We replace $x$ by $x+w$ and $y$ by $y+u$ in (2.1), use (1.3) and rewrite the generating function as:

$$
\begin{gathered}
\frac{1}{1-z\left((1+\lambda t)^{\frac{1}{\lambda}}-1\right)}(1+\lambda t)^{\frac{x+w}{\lambda}}\left(1+\lambda t^{2}\right)^{\frac{y+u}{\lambda}}=\sum_{n=0}^{\infty} H_{n} F_{n, \lambda}(x, y ; z) \frac{t^{n}}{n !} \sum_{s=0}^{\infty} H_{s, \lambda}(w, u) \frac{t^{s}}{s !} \\
=\sum_{n=0}^{\infty} H F_{n, \lambda}(x+w, y+u ; z) \frac{t^{n}}{n !} .
\end{gathered}
$$

Now replacing $n$ by $n-s$ in l.h.s. and comparing the coefficients of $t^{n}$ on both sides, we get the result (3.4).

Theorem 3.3. The following summation formula for degenerate Hermite-Fubini polynomials ${ }_{H} F_{n, \lambda}(x, y ; z)$ holds true:

$$
{ }_{H} F_{n, \lambda}(x, y ; z)=\sum_{r=0}^{n}\left(\begin{array}{c}
n \\
r
\end{array}\right) F_{n-r, \lambda}(x-w ; z) H_{r, \lambda}(w, y) .
$$

Proof. By exploiting the generating function (1.3), we can write equation (2.1) as

$$
\frac{1}{1-z\left((1+\lambda t)^{\frac{1}{\lambda}}-1\right)}(1+\lambda t)^{\frac{x-w}{\lambda}}(1+\lambda t)^{\frac{w}{\lambda}}\left(1+\lambda t^{2}\right)^{\frac{y}{\lambda}}=\sum_{n=0}^{\infty} F_{n, \lambda}(x-w ; z) \frac{t^{n}}{n !} \sum_{r=0}^{\infty} H_{r, \lambda}(w, y) \frac{t^{r}}{r !} .
$$

On replacing $n$ by $n-r$ in above equation, we get

$$
\sum_{n=0}^{\infty}{ }_{H} F_{n, \lambda}(x, y ; z) \frac{t^{n}}{n !}=\sum_{n=0}^{\infty} \sum_{r=0}^{n} F_{n-r, \lambda}(x-w ; z) H_{r, \lambda}(w, y) \frac{t^{n}}{(n-r) ! r !} .
$$

Equating the coefficients of the like powers of $t$ on both sides, we get (3.5).

Theorem 3.4. The following summation formula for degenerate Hermite-Fubini polynomials ${ }_{H} F_{n, \lambda}(x, y ; z)$ holds true:

$$
{ }_{H} F_{n, \lambda}(x+1, y ; z)=\sum_{r=0}^{n}\left(\begin{array}{c}
n \\
r
\end{array}\right){ }_{H} F_{n-r, \lambda}(x, y ; z)\left(\frac{1}{\lambda}\right)_{r} \lambda^{r} .
$$


Proof. Using the generating function (2.1), we have

$$
\begin{gathered}
\sum_{n=0}^{\infty} H_{H} F_{n, \lambda}(x+1, y ; z) \frac{t^{n}}{n !}-\sum_{n=0}^{\infty} H_{n, \lambda} F_{n, y ; z)} \frac{t^{n}}{n !} \\
=\left(\frac{1}{1-1-z\left((1+\lambda t)^{\frac{1}{\lambda}}-1\right.}\right)\left((1+\lambda t)^{\frac{1}{\lambda}}-1\right)(1+\lambda t)^{\frac{x}{\lambda}}\left(1+\lambda t^{2}\right)^{\frac{y}{\lambda}} \\
=\sum_{n=0}^{\infty}{ }_{H} F_{n, \lambda}(x, y ; z) \frac{t^{n}}{n !}\left(\sum_{r=0}^{\infty} \frac{\left(\frac{1}{\lambda}\right)_{r} \lambda^{r} t^{r}}{r !}-1\right) \\
=\sum_{n=0}^{\infty} H F_{n, \lambda}(x, y ; z) \frac{t^{n}}{n !} \sum_{r=0}^{\infty} \frac{\left(\frac{1}{\lambda}\right)_{r} \lambda^{r} t^{r}}{r !}-\sum_{n=0}^{\infty} H F_{n, \lambda}(x, y ; z) \frac{t^{n}}{n !} \\
=\sum_{n=0}^{\infty} \sum_{r=0}^{n}\left(\begin{array}{c}
n \\
r
\end{array}\right)_{H} F_{n-r, \lambda}(x, y ; z)\left(\frac{1}{\lambda}\right)_{r} \lambda^{r} \frac{t^{n}}{n !}-\sum_{n=0}^{\infty}{ }_{H} F_{n, \lambda}(x, y ; z) \frac{t^{n}}{n !} .
\end{gathered}
$$

Finally, equating the coefficients of the like powers of $t$ on both sides, we get (3.6).

\section{Symmetric identities for degenerate Hermite-Fubini polynomials}

In this section, we establish general symmetry identities for the degenerate HermiteFubini polynomials ${ }_{H} F_{n, \lambda}(x, y ; z)$ by applying the generating function $(2.1)$ and $(2.2)$.

Theorem 4.1. Let $x, y, z \in \mathbb{R}$ and $n \geq 0$, then the following identity holds true:

$$
\begin{aligned}
& \sum_{r=0}^{n}\left(\begin{array}{c}
n \\
r
\end{array}\right) b^{r} a_{H}^{n-r} F_{n-r, \lambda}\left(b x, b^{2} y ; z\right)_{H} F_{r, \lambda}\left(a x, a^{2} y ; z\right) \\
= & \sum_{r=0}^{n}\left(\begin{array}{c}
n \\
r
\end{array}\right) a^{r} b^{n-r}{ }_{H} F_{n-r, \lambda}\left(a x, a^{2} y ; z\right)_{H} F_{r, \lambda}\left(b x, b^{2} y ; z\right) .
\end{aligned}
$$

Proof. Start with

$$
A(t)=\frac{(1+\lambda t)^{\frac{a b x}{\lambda}}\left(1+\lambda t^{2}\right)^{\frac{a^{2} b^{2} y}{\lambda}}}{\left(1-z\left((1+\lambda t)^{\frac{a}{\lambda}}-1\right)\right)\left(\left(1-z\left((1+\lambda t)^{\frac{b}{\lambda}}-1\right)\right.\right.} .
$$

Then the expression for $A(t)$ is symmetric in $a$ and $b$ and we can expand $A(t)$ into series in two ways to obtain:

$$
\begin{gathered}
A(t)=\sum_{n=0}^{\infty}{ }_{H} F_{n, \lambda}\left(b x, b^{2} y ; z\right) \frac{(a t)^{n}}{n !} \sum_{r=0}^{\infty}{ }_{H} F_{r, \lambda}\left(a x, a^{2} y ; z\right) \frac{(b t)^{r}}{r !} \\
A(t)=\sum_{n=0}^{\infty}\left(\sum_{r=0}^{n}\left(\begin{array}{c}
n \\
r
\end{array}\right) b^{r} a^{n-r}{ }_{H} F_{n-r, \lambda}\left(b x, b^{2} y ; z\right)_{H} F_{r, \lambda}\left(a x, a^{2} y ; z\right)\right) \frac{t^{n}}{n !} .
\end{gathered}
$$

Similarly, we can show that

$$
\begin{gathered}
A(t)=\sum_{n=0}^{\infty}{ }_{H} F_{n, \lambda}\left(a x, a^{2} y ; z\right) \frac{(b t)^{n}}{n !} \sum_{r=0}^{\infty}{ }_{H} F_{r, \lambda}\left(b x, b^{2} y ; z\right) \frac{(a t)^{r}}{r !} \\
A(t)=\sum_{n=0}^{\infty}\left(\sum_{r=0}^{n}\left(\begin{array}{c}
n \\
r
\end{array}\right) a^{r} b^{n-r}{ }_{H} F_{n-r, \lambda}\left(a x, a^{2} y ; z\right)_{H} F_{r, \lambda}\left(b x, b^{2} y ; z\right)\right) \frac{t^{n}}{n !} .
\end{gathered}
$$

By comparing the coefficients of $\frac{t^{n}}{n !}$ on the right hand sides of the last two equations, we arrive at the desired result (4.1). 
Theorem 4.2. For each pair of integers $a$ and $b$ and all integers and $n \geq 0$, the following identity holds true:

$$
\begin{gathered}
\sum_{k=0}^{n}\left(\begin{array}{c}
n \\
k
\end{array}\right) a^{n-k} b_{H}^{k} F_{n-k, \lambda}\left(b x, b^{2} y ; z\right) \sum_{i=0}^{k}\left(\begin{array}{c}
k \\
i
\end{array}\right) \sigma_{i}\left(\frac{\lambda}{b}, a-1\right) F_{k-i, \lambda}(a u ; z) \\
\sum_{k=0}^{n}\left(\begin{array}{c}
n \\
k
\end{array}\right) b^{n-k} a_{H}^{k} F_{n-k, \lambda}\left(a x, a^{2} y ; z\right) \sum_{i=0}^{k}\left(\begin{array}{c}
k \\
i
\end{array}\right) \sigma_{i}\left(\frac{\lambda}{a}, b-1\right) F_{k-i, \lambda}(b u ; z) .
\end{gathered}
$$

Proof. Let

$$
\begin{gathered}
B(t)=\frac{(1+\lambda t)^{\frac{a b(x+u)}{\lambda}}\left(1+\lambda t^{2}\right)^{\frac{a^{2} b^{2} y}{\lambda}}\left((1+\lambda t)^{\frac{a b}{\lambda}}-1\right)}{\left(1-z\left((1+\lambda t)^{\frac{a}{\lambda}}-1\right)\right)\left(1-z\left((1+\lambda t)^{\frac{b}{\lambda}}-1\right)\left((1+\lambda t)^{\frac{a}{\lambda}}-1\right)\left((1+\lambda t)^{\frac{b}{\lambda}}-1\right)\right.} \\
=\frac{(1+\lambda t)^{\frac{a b x}{\lambda}}\left(1+\lambda t^{2}\right)^{\frac{a^{2} b^{2} y}{\lambda}}}{\left(1-z\left((1+\lambda t)^{\frac{a}{\lambda}}-1\right)\right)} \frac{\left((1+\lambda t)^{\frac{a b}{\lambda}}-1\right)}{\left.(1+\lambda t)^{\frac{b}{\lambda}}-1\right)} \frac{(1+\lambda t)^{\frac{a b u}{\lambda}}}{\left(1-z\left((1+\lambda t)^{\frac{b}{\lambda}}-1\right)\right)} \\
B(t)=\frac{(1+\lambda t)^{\frac{a b x}{\lambda}}\left(1+\lambda t^{2}\right)^{\frac{a^{2} b^{2} y}{\lambda}}}{\left(1-z\left((1+\lambda t)^{\frac{a}{\lambda}}-1\right)\right)}\left(\sum_{i=0}^{\infty} \sigma_{i}\left(\frac{\lambda}{b}, a-1\right) \frac{(b t)^{i}}{i !}\right)\left(\sum_{k=0}^{\infty} F_{k, \lambda}(a u ; z) \frac{(b t)^{k}}{k !}\right) \\
\left(\sum_{n=0}^{\infty} H F_{n, \lambda}\left(b x, b^{2} y ; z\right) \frac{(a t)^{n}}{n !}\right)\left(\sum_{k=0}^{\infty} \sum_{i=0}^{k}\left(\begin{array}{c}
k \\
i
\end{array}\right) b^{k} \sigma_{i}\left(\frac{\lambda}{b}, a-1\right) F_{k-i, \lambda}(a u ; z) \frac{t^{k}}{k !}\right) \\
=\sum_{n=0}^{\infty}\left(\begin{array}{c}
n \\
\left.\sum_{k=0}^{n}\left(\begin{array}{c}
n \\
k
\end{array}\right) a^{n-k} b^{k}{ }_{H} F_{n-k, \lambda}\left(b x, b^{2} y ; z\right) \sum_{i=0}^{k}\left(\begin{array}{c}
k \\
i
\end{array}\right) \sigma_{i}\left(\frac{\lambda}{b}, a-1\right) F_{k-i, \lambda}(a u ; z)\right)
\end{array}\right.
\end{gathered}
$$

On the other hand, we have

$B(t)=\sum_{n=0}^{\infty}\left(\sum_{k=0}^{n}\left(\begin{array}{c}n \\ k\end{array}\right) b^{n-k} a_{H}^{k} F_{n-k, \lambda}\left(a x, a^{2} y ; z\right) \sum_{i=0}^{k}\left(\begin{array}{c}k \\ i\end{array}\right) \sigma_{i}\left(\frac{\lambda}{a}, b-1\right) F_{k-i, \lambda}(b u ; z)\right) \frac{t^{n}}{n !}$.

By comparing the coefficients of $t^{n}$ on the right hand sides of the last two equations, we arrive at the desired result.

\section{References}

[1] Bell, E. T, Exponential polynomials, Ann. of Math., 35(1934), 258-277.

[2] Boyadzhiev, K. N, A series transformation formula and related polynomials, Int. J. Math. Math. Sci., 23(2005), 3849-3866.

[3] Carlitz, L, Degenerate Stirling Bernoulli and Eulerian numbers, Util.Math., 15(1979), 51-88.

[4] Dattoli, G, Lorenzutta, S and Cesarano, C, Finite sums and generalized forms of Bernoulli polynomials, Rendiconti di Mathematica, 19(1999), 385-391.

[5] Graham, R. L, Knuth, D. E, Patashnik, O, Concrete Mathematics, AddisonWesley Publ. Co., New York, 1994.

[6] Gross, O. A, Preferential arrangements, Amer. Math. Monthly, 69(1962), 4-8.

[7] Khan, W. A, A note on degenerate Hermite-poly-Bernoulli numbers and polynomials, J. Classical Anal., 8(1)(2016), 65-76. 
[8] Haroon, H, Khan, W. A, Degenerate Bernoulli numbers and polynomials associated with degenerate Hermite polynomials, Commun. Korean Math. Soc., (accepted), (2018), In Press.

[9] Kim, T, Kim, D. S, Jang, G. W, A note on degenerate Fubini polynomials, Proc. Jangjeon Math. Soc., 20(4)(2017), 521-531.

[10] Kim, D. S, Dolgy, T and Komatsu, D. V, Barnes type degenerate Bernoulli polynomials, Adv. Dtud. Contemp. Math., 25(1)(2015), 121-146.

[11] Kim, T, Barnes type multiple degenerate Bernoulli and Euler polynomials, Appl. Math. Comput., 258(2015), 556-564.

[12] Pathan, M. A and Khan, W. A, Some implicit summation formulas and symmetric identities for the generalized Hermite-Bernoulli polynomials, Mediterr. J. Math., 12(2015), 679-695.

[13] Pathan, M. A and Khan, W. A: A new class of generalized polynomials associated with Hermite and Euler polynomials, Mediterr. J. Math., 13(2016), 913-928.

[14] Srivastava, H. M and Manocha, H. L, A treatise on generating functions, Ellis Horwood Limited. Co., New York, 1984.

[15] Tanny, S. M, On some numbers related to Bell numbers, Canad. Math. Bull., 17(1974), 733-738. 